\title{
A comparative study of PSO, GSA and SCA in parameters optimization of surface grinding process
}

\author{
Teh Muy Shin ${ }^{1}$, Asrul Adam², Amar Faiz Zainal Abidin ${ }^{3}$ \\ ${ }^{1,2}$ Faculty of Manufacturing Engineering, Universiti Malaysia Pahang, Malaysia \\ ${ }^{3}$ Faculty of Engineering Technology, Universiti Teknikal Malaysia Melaka, Malaysia
}

\begin{abstract}
Article Info
Article history:

Received Jan 2, 2019

Revised Mar 11, 2019

Accepted Mar 29, 2019

\section{Keywords:}

Particle swarm optimization Gravitational search algorithm Sine Cosine algorithm Surface grinding process Production costs

ABSTRACT

The selection of parameters in grinding process remains as a crucial role to guarantee that the machined product quality is at the minimum production cost and maximum production rate. Therefore, it is required to utilize more advance and effective optimization methods to obtain the optimum parameters and resulting an improvement on the grinding performance. In this paper, three optimization algorithms which are particle swarm optimization (PSO), gravitational search, and Sine Cosine algorithms are employed to optimize the grinding process parameters that may either reduce the cost, increase the productivity or obtain the finest surface finish and resulting a higher grinding process performance. The efficiency of the three algorithms are evaluated and compared with previous results obtained by other optimization methods on similar studies. The experimental results showed that PSO algorithm achieves better optimization performance in the aspect of convergence rate and accuracy of best solution. Whereas in the comparison of results of previous researchers, the obtained result of PSO proves that it is efficient in solving the complicated mathematical model of surface grinding process with different conditions.
\end{abstract}

Copyright (c) 2019 Institute of Advanced Engineering and Science. All rights reserved.

\section{Corresponding Author:}

Asrul Adam,

Faculty of Manufacturing Engineering,

Universiti Malaysia Pahang,

26600 Pekan, Pahang, Malaysia.

Email: asrul@ump.edu.my

\section{INTRODUCTION}

Grinding process remains as an important conventional machining process that is performed on various kind of surfaces for the shaping and finishing purpose in order to manufacture precision finished products [1]. By comparing with other conventional machining processes like turning and milling, grinding can produce better surface finish with smaller surface roughness. Grinding process involves material removal and surface transmission operation which having disadvantages in terms of resources, materials, energy consumptions and power emissions. The accuracy of the surface tolerance of the grinding process is high and it is capable to machine tough materials. Moreover, the machining process parameter are often decided based on the operator experience and referred the standard technical process parameters table. In any case, it is difficult even a skilled operator to achieve the optimum parameter value for every different grinding process. Consequently, a lot of factors that affect the performance of grinding process had occurred such as poor surface finish, short tool life, spindle speed exceed machine capacity and inconsistent process temperature. Therefore, it is crucial to resolve the grinding application issues by increase the process performance.

In general, the optimization problems can be classified into two categories, which are singleobjective [2] and multi-objective [3,5] problems. By reviewing the previous works with different methods, it can be seen that many researchers have conducted the optimization of grinding process using combinedobjective functions as one single-objective problem to optimize the process parameters [2, 4-10]. This is 
because these optimization methods are having difficulties to expand to real multi objective problems since they are not introduced to solve the multiple ideal solutions. These optimization methods are lacking from the drawback that the decision maker needs to have detailed information to make decision on the ranking of the objective functions. In addition, these basic optimization methods fail to apply in the discontinuous objection function and consequently being modified or combined to form a new approach in order to solve the multiobjective optimization problems. In this study, therefore, the single-objective approach is chosen for the optimization of grinding process.

The motivation of this present works is aimed to evaluate the efficiency of three optimization algorithms; (1) particle swarm optimization (PSO), (2) gravitational search (GSA), and Sine Cosines (SCA) algorithms to the surface grinding process problem. The algorithms are utilized for both rough and finish grinding conditions of the surface grinding process. The findings of this study are analyzed and discussed to suggest the most efficient algorithm for grinding process problem.

\section{MATHEMATICAL FORMULATION OF SURFACE GRINDING PROCESS}

There are three sub-objectives that can be considered in this study which are the production cost, the production rate and the surface finish. The four variables that considered in this paper including the speed of wheel, $V_{r}$, speed of workpiece, $V_{w}$, depth of dressing, Doc, and lead of dressing, $L$. In both rough and finish surface grinding process, the process variables are necessarily to satisfy the standard boundary condition in order to obtain the boundary value. The parameters bounds for the $V_{r}, V_{w}, D o c$, and, $L$, are set to [1000,2023], [10,22.7], [0.01,0.137], [0.01,0.137], respectively. The three sub-objective functions would be separated into two different sections; (1) the rough grinding condition is aimed to minimize the production cost and maximize the production rate, (2) the finish grinding condition is aimed to minimize the production cost and reduce the surface roughness.

\subsection{Objective functions}

The three objective functions that considered in this paper are minimize the production cost $C_{T},(\$ / p c)$, maximize the production rate in terms of workpiece removal parameter $W R P,\left(\mathrm{~mm}^{3} / \mathrm{min} \mathrm{N}\right)$ and minimize the surface roughness $R_{a},(\mu m)$.

\subsection{Production cost}

The total production cost, $C_{T}$ in the surface grinding process include the cost specifically related to the grinding of the part, the cost of non-beneficial time and lastly the cost of material consumption. For the rough grinding process, it is necessarily to minimize the $C_{T}$, considering the various optimization parameter mentioned above is shown in (1) which is introduced by Wen et. al. [2].

$$
\begin{aligned}
& C_{T}=\frac{M_{c}}{60 p}\left(\frac{L_{w}+L_{e}}{V_{\boldsymbol{w}} \times 1000}\right)\left(\frac{b_{w}+b_{e}}{f_{b}}\right)\left(\frac{a_{w}}{a_{p}}+S_{p}+\frac{a_{w} b_{w} L_{w}}{\pi D_{e} b_{s} a_{p} G}\right)+\frac{M c}{60 p}\left(\frac{S_{d}}{V_{r}}+t_{1}\right)+\frac{M_{c} t_{c h}}{60 N_{t}}+ \\
& \frac{M_{c} \pi b_{s} D_{e}}{60 p N_{d} \boldsymbol{L} \boldsymbol{V}_{s} 1000}+C_{s}\left(\frac{a_{w} b_{w} L_{w}}{p G}+\frac{\pi(\boldsymbol{D o c}) b_{s} D_{e}}{p N_{d}}\right)+\frac{C_{d}}{p N_{t d}}
\end{aligned}
$$

Where $M_{c}=$ cost per hour labor and administration, $L_{w}=$ length of workpiece, $L_{e}=$ empty length of grinding, $b_{w}=$ width of workpiece, $b_{e}=$ empty width of grinding, $f_{b}$ cross-feed rate, $a_{w}=$ total thickness of cut, $a_{p}=$ down-feed of grinding, $S_{p}=$ number of spark out grinding, $D_{e}=$ diameter of wheel, $b_{s}=$ width of wheel, $G=$ grinding ratio, $S_{d}=$ distance of wheel idling, $p=$ number of workpieces loaded on the table, $V_{r}=$ speed of wheel idling, $t_{1}=$ time of loading and unloading workpieces, $t_{c h}=$ time of adjusting machine tool, $N_{t}=$ batch size of the workpieces, $N_{d}=$ total number of workpieces to be ground between two dressing, $N_{t d}=$ total number of workpieces to be ground during the life of dresser, $C_{s}=$ cost of wheel per $\mathrm{mm}^{3}$, and $C_{d}=\operatorname{cost}$ of dressing.

\subsection{Production rate}

The production rate in the rough grinding process is represented by the workpieces removal rate, $W R P$. For the rough grinding process, the maximization of production rate in terms of $W R P$ is given by (2) [2].

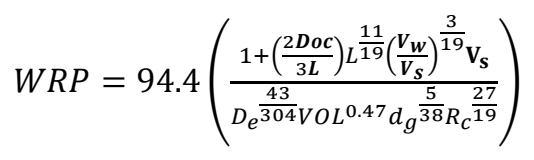


Where VOL is the percentage of wheel bond, $d_{g}$, is the grind size, and $R_{c}$ is the workpiece hardness.

\subsection{Surface finish}

The surface finish of the workpiece is commonly determined to be within a particular value surface roughness, $R_{a}$ that is highly affected by the process parameters and the wheel dressing parameter where the mathematical model is expressed as follow [2],

$$
\begin{aligned}
& R_{a}=0.4587 T_{\text {ave }}{ }^{0.30} 0<T_{\text {ave }}<0.254 \text { else } \\
& R_{a}=0.78667 T_{\text {ave }}{ }^{0.30} 0.254<T_{\text {ave }}<2.54
\end{aligned}
$$

$$
T_{\text {ave }}=12.5 \times 10^{3} \frac{d_{g^{\frac{16}{27}}} a_{p^{\frac{19}{27}}}}{D_{e^{\frac{8}{27}}}^{27}}\left(1+\frac{D o c}{L}\right) L^{\frac{16}{27}}\left(\frac{V_{w}}{V_{s}}\right)^{\frac{16}{27}}
$$

where, $T_{\text {ave }}$ is an average chip thickness during grinding.

\subsection{Constraints}

The major constraints that involve in the optimization problem in surface grinding process are the process and variable constraints. The three major process constraints in both rough and finish grinding process are thermal damage, $U$ wheel wear parameter, $W W P$ and machine tool stiffness. In addition, surface finish will exists as a constraint in the rough grinding process in behalf of a specific surface finish needs to be maintained to obtain the maximum production rate. While in the finish grinding, the production rate exists as a constraint. The grinding process need a high energy per unit volume of material removed. Then, the energy will be converted into heat energy which is thought inside the grinding region. The workpiece will be damaged by the high thermal energy and it prompts to the decreased production rate. The specific energy, $U$ is expressed as (5) [2].

$$
=13.8+\frac{9.64 \times 10^{-4} V_{s}}{a_{p} V_{w}}+\left(6.9 \times 10^{-3}+\frac{2102.4 V_{w}}{D_{e} V_{s}}\right) \times\left(A_{0}+\frac{K_{u} V_{s} L_{w} a_{w}}{V_{\boldsymbol{w}} D_{e}{ }^{1 / 2} a_{p}{ }^{1 / 2}}\right)\left(\frac{V_{s} D_{e}^{1 / 2}}{V_{\boldsymbol{w}} a_{p}^{1 / 2}}\right)
$$

where $A_{0}$ is an initial waer flat area percentage, $\%$ and $K_{u}$ is the wera constant per $m m$. The critical specific energy, $U^{*}$, which resulting the start of thermal damages, is expressed in term of operating parameter as shown in Equation (6) [2].

$$
U^{*}=6.2+1.76 \frac{D_{e}{ }^{1 / 4}}{a_{p}^{3 / 4} V_{w}{ }^{1 / 2}}
$$

Practically, the specific energy must be equal or less than the critical specific energy, $U^{*}$, or else the workpiece burn will be occurred. Based on the relationship between the grinding process parameters and the specific energy, the thermal damage constraint will be specified as Equation (7) [2].

$$
U \leq U^{*}
$$

Wheel wear parameter, $W W P$ is directly related to the grinding process condition and the wheel dressing preceding is shown as Equation (8) [2].

$$
W W P=\left(\frac{K_{a} a_{p} d_{g}{ }^{5 / 38} R_{c}{ }^{27 / 29}}{D_{e}^{1.2 / V O L-43 / 304} V^{0.38}}\right) \times \frac{(1+(\boldsymbol{D o c} / \boldsymbol{L})) L^{27 / 19}\left(\boldsymbol{V}_{s} / \boldsymbol{V}_{\boldsymbol{w}}\right)^{3 / 19} \boldsymbol{V}_{\boldsymbol{w}}}{(1+(2 \boldsymbol{D o c} / 3 L))}
$$

Where $K_{a}=$ constant dependant on coolant and wheel grain type. The wheel wear constraint is obtained from the Equation (2) and (9) is expressed as Equation (9) [2].

$$
\frac{W R P}{W W P} \geq G
$$

The low machining production rate and poor surface quality may cause the chatter results. Chatter avoidance is an obvious constraint in the selection of machining parameters. The relationship between grinding stiffnes, $K_{c}$, wheel wear stiffness, $K_{s}$ and the process parameters is shown in (10) and (11) [2]. 


$$
\begin{aligned}
& K_{c}=\frac{1000 V_{w} f_{b}}{W R P} \\
& K_{S}=\frac{1000 V_{S} f_{b}}{W W P}
\end{aligned}
$$

In order to avoid chatter during machining, the machine tool stiffness constraint, $M S C$ is given as

$$
M S C-\frac{\left|R_{e m}\right|}{K_{m}} \geq 0
$$

Where $M S C=\frac{1}{2 K_{C}}\left(1+\frac{V_{w}}{V_{S} G}\right)+\frac{1}{K_{S}}, \quad K_{m}=$ static machine stiffness and $R_{e m}=$ dynamics machine characteristics.

In the rough grinding condition, it is necessarily to maintain a finest surface finish with the maximum production rate. Therefore, the sub-objective of the rough grinding will be the production rate while the surface finish is a constraint in the rough grinding process. The constraints can be expressed as (13) [2].

$$
R_{a} \leq R_{a}^{*}
$$

While for the finish grinding condition, it is aimed to obtain an optimum surface finish at a particular production rate. Hence, the surface finish is chosen as the sub-objective while the production rate is a constraint. The constraints can be expressed as Equation (14) [2].

$$
W R P \leq W R P^{*}
$$

\subsection{Combined-objective function model}

The sub-objectives of the both rough and finish grinding operation will be normalized to a singleweighted objective as (15) [2]. By combining the sub-objectives, it may effectively overcome the large differences of value between the numerical value and the sub-objectives.

$$
\operatorname{COF}\left(V_{s}, V_{w}, D o c, L\right)=W_{1} \times \frac{C T}{C T^{*}}-W_{2} \times \frac{W R P}{W R P^{*}}+W_{3} \times \frac{R_{a}}{R_{a}{ }^{*}}
$$

Where $C T^{*}=$ expected total production cost limitation and $W_{1}, W_{2}, W_{3}=$ weighting factors for the $C T, W R P$ and $R_{a}$ respectively. For the rough grinding process, the combined objective function model which shown in the Equation (15) is modified as Equation (16) since $W_{3}=0$.

$$
C O F_{R}\left(V_{s}, V_{w}, D o c, L\right)=W_{1} \times \frac{C T}{C T^{*}}-W_{2} \times \frac{W R P}{W R P^{*}}
$$

Where $W_{1}=W_{2}=0.5$ and $W_{3}=0$ based on the analysis and researches on previous papers [2]. While for finish grinding process, the combined objective function model which shown in (15) is modified as (17) since $W_{2}=0$.

$$
C O F_{F}\left(V_{s}, V_{w}, D o c, L\right)=W_{1} \times \frac{C T}{C T^{*}}+W_{3} \times \frac{R_{a}}{R_{a}{ }^{*}}
$$

where $W_{1}=0.3, W_{2}=0$ and $W_{3}=0.7$ based on the analysis and researches on previous papers [2].

\section{OPTIMIZATION ALGORITHM METHODS}

\subsection{Particle swarm optimization}

PSO algorithm is a population-based optimization method that introduced by Kennedy, $\mathbf{J}$ and Eberhart, R. in 1995 [11]. To date, the PSO algorithm has widely been employed in various kind of applications such feature selection [12], feature scaling [13]-[14], and routing in VLSI [15]. The concept of PSO involves the changing the velocity of each particle in each level at each step and weighting the acceleration by the generated random number separately against the ' $p B e s t$ ' and ' $g$ Best' locations. The updates of the particles are expert according to the following (18). 
The equation for the new velocity,$V_{i+1}$ for each particle according to the initial velocity, when the ' $p$ Best' and 'gBest' have achieved is shown in (18).

$$
V_{i+1}=w \times V_{i}+c_{1} \times r_{1} \times\left(p B e s t_{i}-X_{i}\right)+c_{2} \times r_{2} \times\left(g B e s t_{i}-X_{i}\right)
$$

Where $w=$ inertia coefficient,$V_{i}=$ initial velocity, $c_{1}, c_{2}=$ acceleration constant, $p$ Best $_{i}=$ initial $p$ Best $X_{i}=$ individual particle's position, $g$ Best $t_{i}=$ initial $g$ Best and $r_{1}, r_{2}=$ random number from $0 \leq r_{n} \leq 1$ where $n=1$ and 2 . The equation for the personal particle's position, $X_{i+1}$ in solution hyperspace is shown in (19).

$$
X_{i+1}=X_{i}+V_{i+1}
$$

The optimum selection of process parameters of PSO like the $c_{1}$ and $c_{2}$ as well as $w$ is very important for the convergence of methods. By take into account the particle velocity and position at discrete time step, the (18) and (19) are substituted to form the non-homogeneous recurrence relation which is shown in $(20)$.

$$
X_{i+1}=\left(1+w-\emptyset_{1}-\emptyset_{2}\right) X_{i}-w X_{i-1}+\emptyset_{1} \times p \text { Best }_{i}+\emptyset_{2} \times g B e s t_{i}
$$

where $\emptyset_{1}=c_{1} \times r_{1}$ and $\emptyset_{2}=c_{2} \times r_{2}$.

Provide a statement that what is expected, as stated in the "Introduction" chapter can ultimately result in "Results and Discussion" chapter, so there is compatibility. Moreover, it can also be added the prospect of the development of research results and application prospects of further studies into the next (based on result and discussion).

\subsection{Gravitational search algorithm}

The GSA, proposed by Rashedi et. al. in year 2009 [16], is a law of gravity-based optimization method which is also has been used in different applications [17-19]. In GSA, the agents are treated as objects and the performance is calculated by their masses. These objects attract each other by the gravity force which is caused by a global movement of all objects towards the objects with heavier masses. The overall force acquired by all other agents calculates the direction of the agent. There are four major specifications in each mass or agent which including the position, inertia mass, active gravitational mass and lastly passive gravitational mass. The position of the mass is corresponding to the problem solution while the inertia and gravitational masses are determined utilizing a fitness function. Particularly, each mass represent a solution and the inertia and gravitational mass will navigate the algorithm. Theoretically, the masses will be attracted by the heaviest mass that present an optimum solution in the search space. When considering an optimization method with $N$ masses, the position of the $i$ th agents is expressed as (26).

$$
X_{i}=\left(x_{i}{ }^{1}, \ldots x_{i}{ }^{d}, \ldots x_{i}{ }^{n}\right) \quad \text { Where } i=1,2,3, \ldots, N
$$

Where $x_{i}{ }^{d}$ is the position of $i$ th agent in the $d$ th dimension. At certain times ' $t$ ', the force acting on the mass ' $i$ ' from mass ' $j$ ' is expressed as (27).

$$
F_{i j}^{d}=G(t) \frac{M_{p i}(t) \times M_{a j}(t)}{R_{i j}(t)+\varepsilon}\left(x_{j}^{d}(t)-x_{i}^{d}(t)\right)
$$

Where $M_{a j}$ is the active gravitational mass related to agent $j, M_{p i}$ is the passive gravitational mass related to agent $i, G(t)$ is gravitational constant at time $t, \varepsilon$ is a minor constant and $R_{i j}(t)$ is the Euclidean distance between two agents $i$ and $j$ and expressed as (28).

$$
R_{i j}(t)=\left\|X_{i}(t), X_{j}(t)\right\|_{2}
$$

The total force that acts on the agent $i$ in a dimension $d$ be a random weighted sum of $d$ th components of the force that exerted from other agents is expressed as Equation (29) where $\operatorname{rand}_{j}$ is a random number in the interval $[0,1]$.

$$
F_{i}^{d}(t)=\sum_{j=1, j \neq i}^{N} \operatorname{rand}_{j} F_{i j}^{d}(t)
$$


The acceleration of the agent $i$ at time $t$ and the in direction $d$ th, $a_{i}{ }^{d}(t)$ by the law of motion is expressed as Equation (30), where $M_{i i}$ is the inertia weight of the $i$ th agent.

$$
a_{i}^{d}(t)=\frac{F_{i}{ }^{d}(t)}{M_{i i}(t)}
$$

Moreover, the next velocity is considered as a fraction of its current velocity added to its acceleration. The position and velocity is calculated and expressed as (31) and (32), where rand is a random number in the interval $[0,1]$.

$$
\begin{aligned}
& V_{i}^{d}(t+1)=\operatorname{rand}_{i} \times V_{i}^{d}(t)+a_{i}^{d}(t) \\
& X_{i}^{d}(t+1)=X_{i}^{d}(t)+V_{i}^{d}(t+1)
\end{aligned}
$$

The gravitational constant, $G$ is initialized and decreased with time to control the accuracy of search space. Generally, $G$ is a function of the primarily value of $\left(G_{0}\right)$ and time $(t)$ n which is expressed as follow,

$$
G(t)=G\left(G_{0}, t\right)
$$

The fitness evaluation measures the gravitational and inertia masses. A heavier mass indicates higher efficiency of the agent. The better agent will walk slowly and higher attractions. The gravitational and inertia masses are updated as (34), (35) and (36).

$$
\begin{aligned}
& M_{a i}=M_{p i}=M_{i i}=M_{i}, \quad i=1,2, \ldots, N \\
& m_{i}(t)=\frac{\operatorname{fit}_{i}(t)-\operatorname{worst}(t)}{\operatorname{best}(t)-\operatorname{worst}(t)} \\
& M_{i}(t)=\frac{m_{i}(t)}{\sum_{j=1}^{N} m_{j}(t)}
\end{aligned}
$$

$f_{i t}(t)$ indicates the fitness value of the agent $i$ at time $t$. For the optimization problem, the $\operatorname{worst}(t)$ and $\operatorname{best}(t)$ are formulated as (37) and (38).

$$
\begin{aligned}
& \operatorname{best}(t)=\max _{j \in\{1, \ldots N\}} f i t_{i}(t) \\
& \operatorname{worst}(t)=\min _{j \in\{1, \ldots N\}} f i t_{i}(t)
\end{aligned}
$$

In order to perform better compromise between the exploration and exploitation, the numbers of agents are reduced with lapse of time in Equation (29). Therefore, a set of agents with larger mass apply their force to the other is proposed. This policy may decrease the exploration energy and increase the exploitation ability. Initially, the exploration must be used in the algorithm to prevent trapping in a local optimum. By a lapse of interactions, the exploration is necessary to fade out while the exploitation is necessary to fade in. The performance of the GSA will be improved through controlling the exploration and exploitation, only the $K_{\text {best }}$ agents will attract to each other. $K_{\text {best }}$ is the function of time with the primarily value of $K_{0}$ at the starting and reducing with time. Initially, the force is applied on all agents and through a period, $K_{\text {best }}$ is decreased gradually and lastly there will only an agent is applying force to others. The equation (29) is modified as (39).

$$
F_{i}^{d}(t)=\sum_{j=\in K_{\text {best }}, j \neq i}^{N} \operatorname{rand}_{j} F_{i j}{ }^{d}(t)
$$

$K_{\text {best }}$ is the set of the first $K$ agents with the best fitness value and a larger mass.

\subsection{Sine cosines algorithm}

Sine and Cosine Algorithm (SCA) is a population-based optimization method which begin the optimization process with a set of random solutions [20]. The SCA algorithm has been successfully implemented in various engineering problem including parameter optimization of PID controller [21], support vector regression [22], short-term hydrothermal scheduling [23], and other fields at present [24]. 
Basically, SCA is typically divided to two stages which involving the former and exploitation. The position updating equations for both former and exploitation stages are expressed as follow,

$$
\begin{aligned}
& X_{i}^{t+1}=X_{i}^{t}+r_{1} \times \sin \left(r_{2}\right) \times\left|r_{3} p_{i}^{t}-X_{i}^{t}\right| \\
& X_{i}^{t+1}=X_{i}^{t}+r_{1} \times \cos \left(r_{2}\right) \times\left|r_{3} p_{i}^{t}-X_{i i}^{t}\right|
\end{aligned}
$$

where, $X_{i}^{t}$ is the position of the current solution in $i$-thdimension at $t$-th iteration, $r_{1}, r_{2}$ and $r_{3}$ are random numbers, $p_{i}$ is the position of the destination point in $i$-th dimension, and $\|$ represents the absolute value. These two equations are combined and expressed as follow,

$$
X_{i}^{t+1}= \begin{cases}X_{i}^{t}+r_{1} \times \sin \left(r_{2}\right) \times\left|r_{3} p_{i}^{t}-X_{i}^{t}\right|, & r_{4}<0.5 \\ X_{i}^{t}+r_{1} \times \cos \left(r_{2}\right) \times\left|r_{3} p_{i}^{t}-X_{i i}^{t}\right|, & r_{4} \geq 0.5\end{cases}
$$

where, $r_{4}$ is a random number in the interval $[0,1]$.

In SCA, there are four major parameters which are $r_{1}, r_{2}, r_{3}, r_{4}$ as shown in (42). The parameter $r_{1}$ defines the next position's that could be either in the space between the solution and destination or outside it. The parameter $r_{2}$ defines the distance of the movement which supposedly toward or outward the destination. The parameter $r_{3}$ indicates a random weight for the destination in order to stochastically emphasize $\left(r_{3}>1\right)$ or deemphasize $\left(r_{3}<1\right)$ the effect of destination in characterizing thr distance. Lastly, the parameter $r_{4}$ switches equally between the sine and cosine elements in Equation (42).

In order to balance the exploration and exploitation to search for the promising areas of the search space and converge to the global optimum eventually, the range of sine and cosine in Equations (40) to (42) is modified as,

$$
\begin{aligned}
& r_{1}=a-t \frac{a}{T} \\
& r_{2}=2 \pi \times r_{4} \\
& r_{3}=2 \times r_{4}
\end{aligned}
$$

where, $t$ is the current iteration, $T$ is the maximum number of iterations and $a$ is a constant. Over the course of iterations will reduce the range of sine and cosine functions. The SCA explores the search space when the range of the sine and cosine functions are in the interval $[1,2]$ and $[-2,-1]$.

\section{EXPERIMENTAL RESULTS AND DISCUSSIONS}

\subsection{Comparison performance of PSO, GSA, and SCA}

A comparison of performance of the best solution at $K_{\max }=1000$ of PSO (blue line), GSA (red line), and SCA (green line) in both rough and finish grinding conditions are presented in the convergence plots as shown in Figure 1 and Figure 2. Referring to these figures, PSO algorithm shows better optimization performance and the obtained results are more consistent and accurate when compared with GSA and SCA. Generally, GSA and SCA tend to find the global optimum faster than PSO algorithm but the final results are more crucial because they are reflecting the capability of the algorithms to escape from the bad local optima as well as locating a close-global optimum. For this situation, PSO algorithm is able to converge the best solution accurately at maximum iterations of 100, meanwhile for the GSA and SCA, they tend to converge immediately when $K_{\max }$ is large and maximum iterations of 100 as they are commonly used to optimize an immerse range of problems with the fixed small population size. 


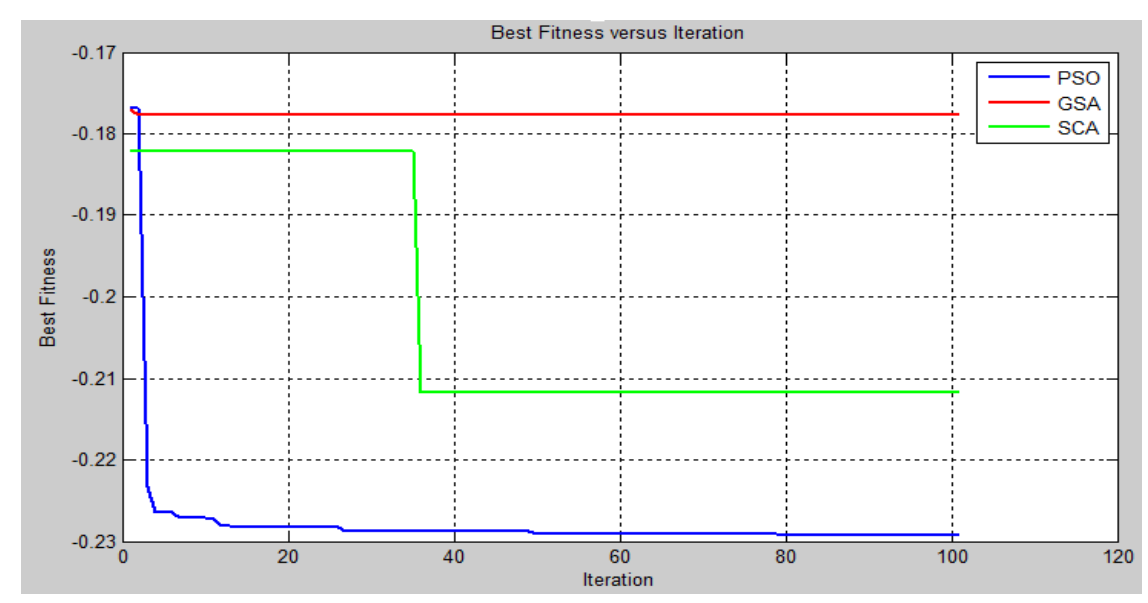

Figure 1. Convergence curve of rough grinding condition

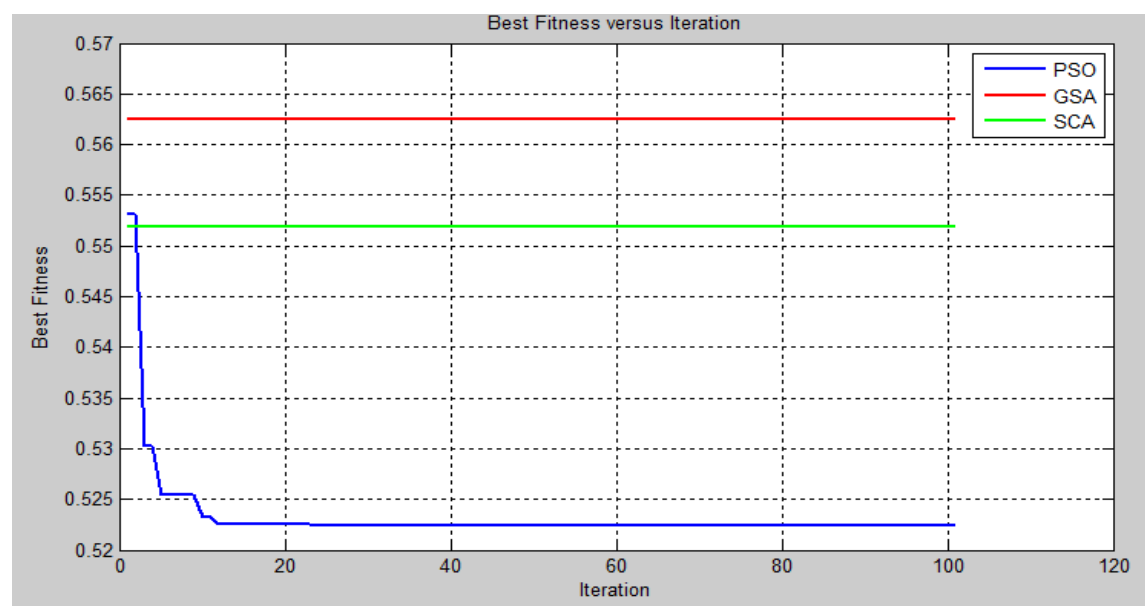

Figure 2. Convergence plot in finish grinding condition

Next, the optimal solution of these PSO, GSA and SCA algorithms are compared to the results of previous researches on similar optimization problem in both rough and finish grinding condition. The smaller value of COF indicates the better optimization. In Table 1, RSFS algorithm shows significant better COF when compared with the optimization algorithms that done by previous researchers as well as PSO algorithm, GSA and SCA. The reasons of RSFS shows better performance may due to the parameters of RSFS are tuned by robust design methodology and providing optimal values for the algorithm. Next, the best solution of PSO algorithm is having similar COF with HPSO, TDEOA and Mod-eDE. The best solution obtained by PSO in this paper shows an improvement of results when compared with the previous research paper that presented by Pawar and Rao (2013) which may influenced by using different value of the parameters and optimzation problem is achieved by the average best solution of 50 independent runs which resulting a more accurate and better results. This proves that PSO algorithm is efficient in solving these optimization problems.

Besides, the comparison results of the finish grinding condition for the surface grinding process are tabulated in Table 2. In Table 2, it can be clearly seen that PSO algorithm shows better optimization performance when compared to most of the optimization algorithms except EP-PSO. Although EP-PSO proposes a superior optimization performance with the minimum $\mathrm{COF}$ value in finish grinding condition, nonetheless its optimization performance in rough grinding condition is just average of these entire optimization algorithms. For this reason, the optimization performance of EP-PSO is slightly inconsistent in solving the optimization problem with different conditions. On the other hand, SCA shows average best solution while the best solution of the GSA is quite unpleasant in the comparison of optimization performance. Briefly, the optimization performance of PSO algorithm is better than GSA and SCA as the solution implemented by PSO algorithm has significant smaller COF value comparing to GSA and SCA. This is because the update operators in PSO algorithm are systematic which allow PSO algorithm to refrain 
trapping in local optima. Moreover, when the population size of the optimization problem is large, PSO algorithm was able to evaluate the best solution within a short time. Meanwhile, GSA and SCA required longer time to evaluate the best solution yet unable to obtain a better solution with smaller value of COF. Subsequently, the obtained result of PSO algorithm proves that it is efficient in solving the complicated mathematical model of the surface grinding process in both rough and finish grinding condition.

Table 1. Comparison results of various optimization algorithms in rough grinding condition

\begin{tabular}{|c|c|c|c|c|c|c|c|c|}
\hline Method & Vs & $\mathrm{VW}_{\mathrm{w}}$ & Doc & $\mathrm{L}$ & CT & WRP & $\mathrm{Ra}$ & $\mathrm{COF}$ \\
\hline RSFS [4] & 2023 & 13.150 & 0.0740 & 0.137 & 7.273 & 23.716 & 1.7999 & -0.2293 \\
\hline HPSO [9] & 2023 & 13.290 & 0.0730 & 0.137 & 7.236 & 23.641 & 1.7999 & -0.2292 \\
\hline TDEOA [8] & 2023 & 13.170 & 0.0740 & 0.167 & 7.267 & 23.701 & 1.8000 & -0.2292 \\
\hline PSO [This study] & 2023 & 13.115 & 0.0745 & 0.137 & 7.281 & 23.730 & 1.7999 & -0.2292 \\
\hline Mod- $\varepsilon D E[6]$ & 2023 & 12.950 & 0.0760 & 0.137 & 7.261 & 23.690 & 1.7999 & -0.2292 \\
\hline HCL-PSO [4] & 2023 & 13.860 & 0.0680 & 0.137 & 7.096 & 23.353 & 1.7996 & -0.2290 \\
\hline EP-PSO [5] & 2023 & 10.490 & 0.1160 & 0.082 & 7.400 & 24.300 & 1.8000 & -0.2233 \\
\hline SCA [This study] & 2023 & 10.000 & 0.1040 & 0.137 & 8.311 & 25.090 & 1.7587 & -0.2117 \\
\hline MPEDE [4] & 1991 & 15.110 & 0.0580 & 0.128 & 6.828 & 22.024 & 1.7923 & -0.2092 \\
\hline GSA [This study] & 1983 & 10.708 & 0.0932 & 0.137 & 6.978 & 24.108 & 1.7696 & -0.2019 \\
\hline GA [7] & 1998 & 11.300 & 0.1010 & 0.065 & 7.863 & 22.256 & 1.7747 & -0.1632 \\
\hline $\mathrm{QP}[2]$ & 2000 & 19.960 & 0.0550 & 0.044 & 6.204 & 17.506 & 1.7477 & -0.1275 \\
\hline \multicolumn{9}{|c|}{ Rough Grinding Condition: $w_{1}=0.5, w_{2}=0.5, w_{3}=0\left(a_{w}=0.1 \mathrm{~mm}, a_{p}=0.050 \mathrm{~mm} / \mathrm{pass}, R_{a} \leq 1.8\right)$} \\
\hline
\end{tabular}

Table 2. Comparison results of the performance of various optimization algorithms in finish

\begin{tabular}{|c|c|c|c|c|c|c|c|c|}
\hline Method & Vs & Vw & Doc & $\mathrm{L}$ & CT & WRP & $\mathrm{Ra}$ & $\mathrm{COF}$ \\
\hline EP-PSO [5] & 2023 & 20.22 & 0.015 & 0.137 & 6.700 & 20.000 & 0.7600 & 0.5040 \\
\hline PSO [This study] & 2023 & 22.70 & 0.0109 & 0.137 & 7.13 & 20.000 & 0.7934 & 0.5224 \\
\hline RSFS [4] & 2023 & 22.70 & 0.0109 & 0.137 & 7.130 & 20.000 & 0.7934 & 0.5224 \\
\hline HPSO [16] & 2023 & 22.70 & 0.0109 & 0.137 & 7.130 & 20.000 & 0.7934 & 0.5224 \\
\hline TDEOA [8] & 2023 & 22.66 & 0.011 & 0.137 & 7.136 & 20.001 & 0.7931 & 0.5225 \\
\hline Mod-eDE [6] & 2023 & 22.70 & 0.011 & 0.137 & 7.132 & 20.005 & 0.7935 & 0.5225 \\
\hline MPEDE [4] & 2023 & 22.14 & 0.012 & 0.137 & 7.212 & 20.020 & 0.7891 & 0.5232 \\
\hline $\mathrm{ACO}[10]$ & 2023 & 19.36 & 0.019 & 0.134 & 7.694 & 20.012 & 0.7644 & 0.5281 \\
\hline SCA [This study] & 2017 & 17.34 & 0.041 & 0.115 & 8.207 & 20.334 & 0.7756 & 0.5332 \\
\hline GA [7] & 1986 & 21.40 & 0.024 & 0.136 & 7.371 & 20.616 & 0.8268 & 0.5427 \\
\hline HCL-PSO [4] & 1965 & 18.74 & 0.0338 & 0.127 & 7.875 & 20.260 & 0.8037 & 0.5488 \\
\hline GSA [This study] & 1944 & 19.10 & 0.036 & 0.127 & 7.361 & 20.259 & 0.8196 & 0.5531 \\
\hline $\mathrm{QP}[2]$ & 2000 & 19.99 & 0.052 & 0.091 & 7.719 & 20.088 & 0.8309 & 0.5547 \\
\hline \multicolumn{9}{|c|}{ Finish Grinding Condition: $w_{1}=0.3, w_{2}=0, w_{3}=0.7\left(a_{w}=0.055 \mathrm{~mm} \mathrm{n}:, a_{p}=0.0105 \mathrm{~mm} /\right.$ pass, WRP $\left.\geq 20 \frac{\mathrm{mm}^{3}}{\mathrm{~mm}-\mathrm{N}}\right)$} \\
\hline
\end{tabular}

\section{CONCLUSIONS}

In this paper, PSO algorithm is applied to optimize the surface grinding process parameter in both rough and finish grinding condition. The optimal value of the decision variables which including $V_{s}, V_{w}, D o c$ and $L$ were obtained by using the mathematical model with the purpose of reducing the production cost and increasing the production rate as well as improving the surface finish. An experiment had conducted to evaluate the efficiency of these proposed algorithms as well as obtain results that are more reliable According to the obtained results from the experiment, it can be observed that PSO algorithm shows superior results when compared to the proposed GSA and SCA. Thus, the experimental results were compared with previous studies and successful evaluated the performance of PSO algorithm. Throughout the comparison, it can be found that the obtained results of proposed PSO algorithms shows better optimization performance when compared to most of the optimization algorithms except EP-PSO. Moreover, the obtained result of this paper is showing significant improvement when compared with the similar study of PSO algorithm by Pawar and Rao [25].

Furthermore, the optimization performance of PSO algorithm in aspects of convergence rate and accuracy of the best solution was successfully achieved. By comparing with GSA and SCA, PSO algorithm is able to achieve good convergence time of the best solution with higher accuracy. Additionally, the maximum iteration of 100 is sufficient for PSO algorithm to convergence accurate and consistent best solution and it meant it could be easily redesign to optimize various process parameters of different conventional machining processes like turning, milling and so on. Subsequently, the obtained result of PSO algorithm proves that it is efficient in solving the complicated multi objective optimization model of the surface grinding process in both rough and finish grinding condition. 


\section{ACKNOWLEDGEMENTS}

This research is funded by Internal UMP Research Grant (RDU180394) that awarded by Ministry of Education Malaysia to Universiti Malaysia Pahang (UMP). Also thanks to Faculty of Manufacturing Engineering, UMP for supporting this research work through the utilization of faculty's equipments.

\section{REFERENCES}

[1] Tönshoff, H. K., Karpuschewski, B., Mandrysch, T., \& Inasaki, I., "Grinding process achievements and their consequences on machine tools challenges and opportunities," CIRP Annals-Manufacturing Technology, vol. 47, no. 2, pp. 651-668, 1998.

[2] Wen, X.M., A.A.O. Tay, A.Y.C. Nee, "Micro-Computer-Based Optimization of the Surface Grinding Process," Journal of Materials Processing Technology, vol. 29, no. 1-3, pp. 75-90, 1992.

[3] Janardhan, M., "An Integrated Evaluation Approach for Modelling and Optimization of Surface Grinding Process Parameters," Materials Today: Proceedings, vol 2, nom. 4-5, pp. 1622-1633, 2015.

[4] Khalilpourazari, S., \& Khalilpourazary, S., "A lexicographic weighted Tchebycheff approach for multi-constrained multi-objective optimization of the surface grinding process," Engineering Optimization, vol. 49, no. 5, pp. 878895, 2017.

[5] X. Lin and H. Li, "Enhanced Pareto Particle Swarm Approach for Multi-Objective Optimization of Surface Grinding Process," 2008 Second International Symposium on Intelligent Information Technology Application, Shanghai, 2008, pp. 618-623.

[6] Rana, P., \& Lalwani, D. I., "Parameters optimization of surface grinding process using Modified $\varepsilon$ constrained Differential Evolution," Materials Today: Proceedings, vol. 4, no. 9, pp. 10104-10108, 2017.

[7] Saravanan, R., Asokan, P., \& Sachidanandam, M., "A multi-objective genetic algorithm (GA) approach for optimization of surface grinding operations," International Journal of Machine Tools and Manufacture, vol. 42, no. 12, pp. 1327-1334, 2002.

[8] J. Tsai, K. Lee and J. Chou, "Robust Evolutionary Optimal Tolerance Design for Machining Variables of Surface Grinding Process," in IEEE Transactions on Industrial Informatics, vol. 10, no. 1, pp. 301-312, Feb. 2014.

[9] Zhang, G., Liu, M., Li, J., Ming, W., Shao, X., \& Huang, Y., "Multi-objective optimization for surface grinding process using a hybrid particle swarm optimization algorithm," The International Journal of Advanced Manufacturing Technology, vol. 71, no. 9, pp. 1861-1872, April 2014.

[10] Baskar, N., Saravanan, R., Asokan, P., \& Prabhaharan, G., "Ants colony algorithm approach for multi-objective optimisation of surface grinding operations," The International Journal of Advanced Manufacturing Technology, vol. 23, no. 5-6, pp. 311-317, 2004.

[11] Kennedy, J., \& Eberhart, R., "Particle Swarm Optimization," Paper presented at the Proceedings of the IEEE International Conference on Neural Networks (ICW), Perth, Western Australia, 1995.

[12] Asrul Adam, Mohd Ibrahim Shapiai, Mohd Zaidi Mohd Tumari, Mohd Saberi Mohamad, and Marizan Mubin, "Feature Selection and Classifier Parameters Estimation for EEG Signals Peak Detection Using Particle Swarm Optimization," The Scientific World Journal, vol. 2014, pp. 1-13, 2014.

[13] K. G. Li, M. I. Shapiai, A. Adam and Z. Ibrahim, "Feature scaling for EEG human concentration using particle swarm optimization," 2016 8th International Conference on Information Technology and Electrical Engineering (ICITEE), Yogyakarta, 2016, pp. 1-6.

[14] Adam, A., Ibrahim, Z., Mokhtar, N., Shapiai, M. I., Cumming, P., \& Mubin, M., "Improving EEG signal peak detection using feature weight learning of a neural network with random weights for eye event-related applications," Sādhanā, vol. 42, no. 5, pp. 641-653, May 2017.

[15] Z. M. Yusof et al., "A Two-Step Binary Particle Swarm Optimization Approach for Routing in VLSI," ICIC Express Letters," vol. 6., no. 3, pp. 771-776, 2012.

[16] Rashedi, E., Nezamabadi-pour, H., \& Saryazdi, S, "GSA: A Gravitational Search Algorithm. Information Sciences," Information Sciences, vol. 179, no. 13, pp. 2232-2248, 2009.

[17] A. Adam, N. Mokhtar, M. Mubin, Z. Ibrahim, M. Z. M. Tumari and M. I. Shapiai, "Feature Selection and Classifier Parameter Estimation for EEG Signal Peak Detection Using Gravitational Search Algorithm," 2014 4th International Conference on Artificial Intelligence with Applications in Engineering and Technology, Kota Kinabalu, 2014, pp. 103-108.

[18] K. Z. M. Azmi, D. Pebrianti, Z. Ibrahim, S. Sudin and S. W. Nawawi, "Simultaneous Computation of Model Order and Parameter Estimation for System Identification Based on Gravitational Search Algorithm," 2015 6th International Conference on Intelligent Systems, Modelling and Simulation, Kuala Lumpur, 2015, pp. 135-140.

[19] Ibrahim, I., Ibrahim, Z., Ahmad, H., Yusof, Z. M., Shapiai, M. I., Nawawi, S. W., \& Mubin, M., "An Assembly Sequence Planning Approach with Binary Gravitational Search Algorithm," In SoMeT, August 2014, pp. 179-193.

[20] Mirjalili, S., "SCA: A Sine Cosine Algorithm for solving optimization problems," Knowledge-Based Systems, vol. 96, pp. 120-133, 2016.

[21] Shashikant, Shaw B., "Comparison of SCA-Optimized PID and P\&O-Based MPPT for an Off-grid Fuel Cell System," In: Nayak J., Abraham A., Krishna B., Chandra Sekhar G., Das A. (eds) Soft Computing in Data Analytics. Advances in Intelligent Systems and Computing, vol 758, 2019, Springer, Singapore

[22] S. Li, H. Fang, and X. Liu, Parameter optimization of support vector regression based on sine cosine algorithm," Expert Systems with Applications, vol. 91, pp. 63-77, 2018. 
[23] S. Das, A. Bhattacharya, and A. K. Chakraborty, Solution of short-term hydrothermal scheduling using sine cosine algorithm," Soft Computing, vol. 6, pp. 1-19, .

[24] Bidyadhar Rout, B.B. Pati, and S. Panda, Modified SCA Algorithm for SSSC Damping Controller Design in Power System," ECTI Transactions on Electrical Engineering, Electronics, and Communications, vol.16, no.1, pp. 46-63, 2018.

[25] Pawar, P. J., \& Rao, R. V., "Parameter optimization of machining processes using teaching-learning-based optimization algorithm," The International Journal of Advanced Manufacturing Technology, vol. 67, no. 5-8, pp. 995-1006, 2013

\section{BIOGRAPHIES OF AUTHORS}

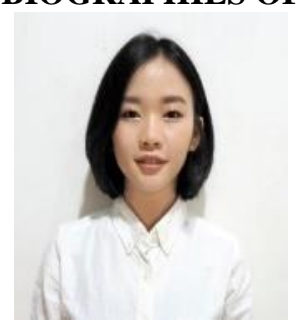

Teh Muy Shin received his first degree, B.Eng. degree in Manufacturing Engineering from Universiti Malaysia Pahang, Malaysia in 2018. His research interest involves parameters optimization on machining processes.

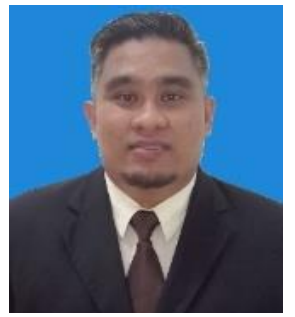

Asrul Adam received the B.Eng. degree and M.Eng. degree in Electrical-Mechatronics and Electrical Engineering (Computational Intelligence) from Universiti Teknologi Malaysia (UTM), Malaysia, in 2009 and 2012, respectively. He received his PhD degree in Signal and System from University of Malaya, Malaysia in 2017. Currently, he is a senior lecturer at the Faculty of Manufacturing Engineering, Universiti Malaysia Pahang, Pahang, Malaysia where he is conducting teaching activities in the areas of electronics and mechatronics engineering. Asrul Adam's research activities concern on the areas of biomedical signals processing, artificial intelligence, brain computer interface, machine learning, and optimization. He has published 15 Scopus/ISI-WoS indexed journals, 20 international conferences, and one book chapter. Asrul Adam recently has 110 total citation with 6 h-index and 234 total citation with 9 h-index based on SCOPUS and Google Scholar, respectively.

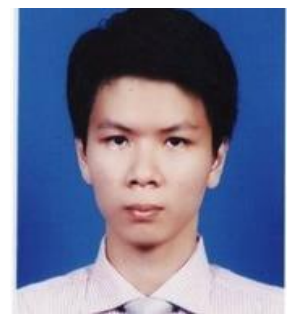

Amar Faiz Zainal Abidin received his first degree, B.Eng. (Hons) in Electrical and Electronics Engineering from The University of Nottingham, Malaysia and M.Eng. from Universiti Teknologi Malaysia (UTM). He is currently attached as an academic staff with Universiti Teknikal Malaysia Melaka (UTeM). His research interests include multiobjective optimisation and nature-inspired optimization algorithms. 